\title{
Monitoreo de medios y democratización en América Latina. La participación ciudadana en la vigilancia de la función informativa de los medios de comunicación de masas
}

\author{
Francisco de Jesús Aceves GonzÁlez*
}

En el presente trabajo se ofrece, en primera instancia, una panorámica sobre la emergencia y desarrollo de esta práctica de observación ciudadana en América Latina; en segundo lugar, se analiza el impacto que dicha observación ha tenido en las políticas informativas de los medios, específicamente en el caso mexicano y, por último, se plantean algunas tareas que la ciudadanía debe afrontar en la defensa de su derecho a una información confiable y en la vigilancia de la actividad informativa de los medios en los procesos políticos.
This article shows, at a first resort, a vantage view about the emergence and development of this citizenship observation practice in Latin America; secondly, the impact so far of that practice on informative politics of the media is analyzed, with the Mexican treated as a specific case, and finally a set up of some tasks that citizens must face up to in defense of their right to a reliable information for watchfulness of the informative activities of the media in politics processes.

* Universidad de Guadalajara. faceves@cencar.udg.mx 
En las sociedades contemporáneas la configuración del espacio público pasa necesariamente por los medios de comunicación masiva. Su centralidad en los procesos de comunicación política, específicamente en los procesos electorales, su capacidad para establecer la agenda de los asuntos públicos, convierten a los medios en un elemento fundamental -más allá de sus características instrumentales- en la construcción democrática. Empero, los intereses particulares de éstos, derivados tanto de sus determinaciones histórico-estructurales como de sus políticas editoriales, se confrontan de manera permanente con los intereses ciudadanos que demandan de ellos el suministro de una información extensa y confiable sobre los asuntos públicos, y con base en la cual se tomen las decisiones que resulten convenientes.

Resulta evidente que los criterios de noticiabilidad que favorecen la "espectacularización de lo político" contrastan con las necesidades informativas de sus usuarios. En este contexto, la emergencia de grupos civiles, que propugnan por establecer una mayor vigilancia a los contenidos informativos suministrados por los medios, constituye una tendencia creciente.

En los países latinoamericanos, un aspecto importante en la transición democrática que se experimenta en sus procesos electorales, ha tenido como factor sustancial la intervención de la sociedad civil en la observación de los procesos electorales. Ésta no se circunscribe a la jornada comicial sino que incluye la vigilancia de las condiciones en que se desarrolla la contienda electoral, particularmente en lo que se refiere a la cobertura informativa que los medios masivos otorgan a las campañas políticas de los candidatos.

En este trabajo se ofrece, en primera instancia, una panorámica sobre la emergencia y desarrollo de esta práctica de observación ciudadana en América Latina; en segundo lugar, se analiza el impacto que dicha observación ha tenido en las políticas informativas de los medios, específicamente en el caso mexicano y, por último, se plantean algunas tareas que la ciudadanía debe afrontar en la defensa de su derecho a una información confiable y en la vigilancia de la actividad informativa de los medios en los procesos políticos. 


\section{SuRgimiENTO Y DESARROLLO DEL MONITOREO DE MEDIOS EN AmÉRICA LATINA}

Aunque el análisis de la cobertura informativa de los medios de comunicación en los procesos electorales se encuentra presente en los trabajos seminales sobre la influencia de los medios en dichos procesos, y aunque el análisis de contenido se constituyó desde su surgimiento en una técnica de investigación utilizada en forma intensiva por las instituciones gubernamentales y por empresas de mercadotecnia, su descubrimiento y utilización por parte de organizaciones de la sociedad civil, como instrumento de análisis para observar y evaluar el comportamiento informativo de los medios masivos sobre determinados acontecimientos sociales, entre ellos, la cobertura informativa que los medios otorgan a los diversos partidos y candidatos en los procesos electorales, es muy reciente.

De esta manera, el análisis de contenido del comportamiento informativo de los medios se convirtió, de instrumento de investigación académica, mercadoténica y de planificación institucional, en herramienta de vigilancia de la sociedad civil, en una práctica de la acción ciudadana: el monitoreo de medios.

Una definición concisa establece que, "en su nivel más básico, el monitoreo de medios para la promoción de elecciones democráticas consiste en una observación independiente e imparcial del comportamiento de los medios masivos durante el periodo electoral". Entre los aspectos principales hacia los cuales enfocar la observación, se destacan:

1. Cómo actúa el gobierno para asegurar el derecho de los medios informativos para recoger y distribuir información e ideas.

2. Cómo actúan el gobierno y los medios para garantizar el acceso de los partidos políticos y sus candidatos, de tal manera que ellos puedan comunicarse efectiva y directamente con el público durante el periodo de las campañas electorales.

3. Cómo actúan el gobierno y los medios para asegurar una exacta y equitativa cobertura informativa de los partidos políticos y de los candidatos en los programas noticiosos y en los reportajes informativos.

4. Cómo actúan el gobierno y los medios para educar al electorado sobre el cómo y el por qué votar (Norris y Merloe 2002). 
En América Latina la emergencia de este tipo de prácticas coincide con las profundas transformaciones políticas experimentadas por diversos países en las últimas décadas del siglo pasado. Específicamente, en el deterioro de los regímenes autoritarios, tanto militares (Argentina, Chile) como civiles (México), ante el arribo de una fuerte corriente democratizadora que -con dificultades y logros, avances y retrocesosse ha venido consolidando en el continente.

El objetivo común de estos grupos ha sido pugnar por el establecimiento de condiciones de competencia que garanticen la participación de todas las corrientes políticas, en los procesos electorales, en términos de equidad y transparencia, en lo relativo al ejercicio de sus actividades proselitistas. Un aspecto fundamental en la contienda política, en estos tiempos caracterizados por la mediatización del espacio público, radica en la posibilidad de los actores políticos de acceder a los medios y, en forma particular, en el tratamiento informativo que los mismos realizan sobre las campañas.

En este contexto, el monitoreo de medios se ha convertido en un valioso instrumento en manos de organizaciones democráticas, para impulsar y promover el desarrollo de procesos electorales democráticos, a partir de la existencia de ciudadanos mejor informados, mediante la vigilancia del comportamiento informativo de los medios, a los cuales se demanda la observancia de los principios de la objetividad informativa y el tratamiento equitativo de los candidatos en la cobertura de sus campañas.

En el caso de México, aunque el antecedente en este tipo de análisis fue el trabajo pionero que Petra Secanella (1983) realizó sobre la cobertura informativa de las campañas políticas de algunos diarios nacionales en las elecciones presidenciales de 1970, 1976 y 1982, y aunque las controversiales elecciones de 1988 fueron objeto de investigación de varios estudiosos (De Garay y Moya 1989; Fregoso 1990; Trejo Delarbre 1990) y estos trabajos documentaron y denunciaron el tratamiento profundamente inequitativo que los medios habían otorgado a los candidatos de la oposición, la publicación de sus resultados ocurrió varios meses después de concluido el proceso electoral. En este sentido, sus importantes hallazgos acerca del comportamiento de los medios carecían de la oportunidad informativa que hubieran tenido en el marco de las contiendas electorales.

Empero, fue cuando la Academia Mexicana de Derechos Humanos (AMDH) desarrolló en 1992 una metodología para realizar análisis de con- 
tenido electoral de medios de comunicación (Acosta Valverde 1997), que la práctica de monitoreo de medios se estableció como un instrumento para impulsar el desarrollo de elecciones transparentes y democráticas.

En las elecciones presidenciales de 1994 se constituyó Alianza Cívica, organismo formado por diversos grupos de la sociedad civil (Academia Mexicana de Derechos Humanos, Acuerdo por la Democracia, Convergencia de Organismos Civiles por la Democracia, Consejo para la Democracia, Fundación Arturo Rosenbleuth, Instituto Superior de Cultura Democrática, y Movimiento Ciudadano por la Democracia), con el objetivo de realizar una observación integral del proceso electoral. Entre las diferentes acciones derivadas de esta observación, se encontraba en un sitio destacado el monitoreo de medios de comunicación (Acosta Valverde y Parra Rosales 1995).

El monitoreo, desarrollado en diversos estados de la república, consistió en un análisis cuantitativo de periódicos y programas noticiosos de radio y televisión, que buscaba "ser una acción propositiva ante la ausencia de mecanismos para garantizar la equidad en la cobertura de los medios de comunicación". Las actividades realizadas fueron:

- Investigación de la cobertura de las campañas en diferentes medios de prensa, radio y TV.

- Información a la opinión pública sobre coberturas objetivas e imparciales o diferencias y parcialidad de diferentes medios de comunicación.

- Denuncia ante las autoridades electorales de los patrones de inequidad y falta de objetividad de los medios de comunicación que caigan en esas prácticas (Álvarez Icaza s/f).

Empero, para 1994 el Instituto Federal Electoral, organismo público, autónomo, responsable de organizar las elecciones federales, en cuyo seno el peso y la influencia de los consejeros ciudadanos era mayoritario, se encontraba particularmente sensible ante la evidente inequidad que los medios otorgaban a las campañas políticas. De ahí, tomó la determinación de realizar un monitoreo, con base en una muestra de ocho telenoticieros y seis radionoticieros sobre la cobertura informativa de la campañas de los candidatos a la presidencia.

Dos años después, en la reforma de la legislación electoral realizada en 1996, quedó establecido en el Artículo 48, inciso 12 del Código Federal de Instituciones y Procedimientos Electorales (COFIPE) que "la 
Comisión de Radiodifusión realizará monitoreos muestrales de los tiempos de transmisión sobre las campañas de los partidos políticos en los espacios noticiosos de los medios de comunicación, para informar al Consejo General".

Para las elecciones de 2000, el monitoreo de medios electrónicos realizado por el IFE abarcó 210 noticieros (126 de radio y 84 de televisión) a nivel nacional. Este hecho, aunado a la transparencia y profesionalismo con que se realiza la actividad, así como la convicción expresada por alguno de sus miembros al reconocer que la práctica del monitoreo "ha probado su utilidad como herramienta de escrutinio del comportamiento de los medios que son cada vez más el espacio privilegiado del desarrollo y las campañas políticas", ${ }^{1}$ ha venido a complementar, en forma exitosa debido a la extensión y calidad del trabajo, a las prácticas realizadas por los organismos de ciudadanos que propugnan por la democratización de los procesos electorales. En efecto, la participación de organismos de la sociedad civil en el monitoreo de medios continúa. En las elecciones de 2000 el Observatorio Ciudadano de Medios, formado por Alianza Cívica, la Academia Mexicana de Derechos Humanos y el Consejo de Educación de Adultos de América Latina, definió una estrategia denominada "Ya los vimos y los vamos a vigilar", consistente en el monitoreo de cuatro noticieros de televisión de difusión nacional, subrayando en su análisis en especial los aspectos cualitativos de la cobertura informativa.

Sin embargo, habría que apuntar que, si bien los medios audiovisuales desempeñan un papel relevante en la construcción de las percepciones políticas de la audiencia, la prensa escrita representa un importante espacio de debate político, en la medida en que, además de la permanencia y profundidad que su formato permite a las informaciones políticas, sigue siendo el espacio de debate privilegiado por las élites políticas. En este sentido, la ausencia de un monitoreo sobre la prensa escrita constituye un vacío fundamental, que provoca que la observación del comportamiento de los medios realizada por el IFE a nivel nacional, quede incompleta. Resulta interesante apuntar que quienes han incidido en suplir esta carencia, no han sido organismos ciudadanos, sino investigadores universitarios que desde las instancias académicas estudian el papel de los medios en los procesos electorales.

No obstante que en lo relativo al ejercicio de monitoreo de medios en los procesos electorales de carácter federal, el caso de México resulta

\footnotetext{
${ }^{1}$ Consejera Jacqueline Peschard, Acta de sesiones del Consejo General del Instituto Federal Electoral del 3 de junio de 1997.
} 
excepcional, no sólo en el ámbito latinoamericano sino mundial, en lo relativo a los procesos electorales de carácter estatal (gubernatura, presidencia municipal y diputado local) la realización de esta actividad presenta profundas desigualdades.

Sólo un puñado (Jalisco, Nuevo León, Estado de México) de los 32 estados han incorporado en sus respectivas legislaciones electorales, la obligación por parte de las autoridades de realizar el monitoreo de medios sobre la cobertura de la campañas electorales y de difundir los resultados.

Así, en el artículo 132, fracción 38 de la Ley Electoral del Estado de Jalisco, se establecece como atribución de su Consejo Electoral "realizar periódicamente muestreos sobre la cobertura que los medios de comunicación realicen sobre las campañas políticas, debiendo publicar los resultados mensualmente". Por su parte, el Código Electoral del Estado de México en su artículo 66 párrafos cuarto y último, determina que el Consejo General

[...] realizará monitoreos cuantitativos y cualitativos y el seguimiento de notas informativas en medios de comunicación impresos y electrónicos a través de una comisión e informará periódicamente al mismo sobre los resultados de tales monitoreos y seguimiento, que serán quincenales en tiempo de proceso electoral. Los informes referidos deberán contener una valoración de la actuación de los medios de comunicación monitoreados, así como las recomendaciones que se estimen conducentes. El Consejo General publicará los resultados de esta actividad en los principales diarios con circulación en el Estado.

Asimismo, el artículo 81, fracción 26 de la Ley Electoral del Estado de Nuevo León, señala como obligación de la Comisión Estatal Electoral la realización de

[...] monitoreos de los medios de comunicación referentes a noticias de prensa, radio, televisión y en general todo medio de comunicación masivo, para conocer el espacio y tiempo dedicado a la cobertura informativa de los partidos políticos y de sus candidatos; el resultado de dicho monitoreo será dado a conocer por lo menos una vez al mes a los partidos políticos, mediante informe escrito y a la opinión pública, por medio de la publicación trimestral del mismo en algún periódico de los que tengan mayor circulación en la entidad. 
En el resto de las entidades, la práctica del monitoreo en los procesos estatales es casi inexistente. Entre las escasas experiencias en este rubro, destacan las realizadas en Yucatán en las elecciones de 2000 por el Frente Cívico Familiar (FCF). ${ }^{2}$ En ese mismo año, en el contexto del proceso electoral para la gubernatura en Chiapas, un grupo de profesores de la Universidad Autónoma de Chiapas, con el auspicio del Consejo Estatal Electoral de Chiapas realizó el primer monitoreo de prensa escrita en el estado (Matus López 2001). Coincidentemente, en el marco de las elecciones estatales celebradas en octubre en Tabasco, tres asociaciones civiles: Alianza Cívica de Tabasco, Mujeres Solidarias en Acción Social (musas) y el Comité de Derechos Humanos de Tabasco (Codehutab) realizaron el monitoreo de cuatro diarios locales.

En el sur del continente, los esfuerzos pioneros en la observación ciudadana del comportamiento de los medios en los procesos electorales, se encuentra en el marco de las actividades desarrolladas por un grupo de profesores universitarios que con el objetivo de "Contribuir a que los procesos electorales que se lleven a cabo en el Perú sean democráticos y transparentes y representen la real voluntad ciudadana", fundaron en julio de 1994 la asociación civil TRANSPARENCIA: un "movimiento cívico, pedagógico, con voluntarios a nivel nacional sin afiliación política".

Bajo el lema de "Sin vigilancia no hay democracia", esta organización ha impulsado un conjunto de actividades entre las que destaca la "organización de sistemas independientes de servicios de información electoral, conteos paralelos y observación electoral, con la finalidad de una mayor y mejor participación de la ciudadanía y contribuir a la transparencia de los procesos electorales". Específicamente, el rubro de observación electoral ha establecido, entre otras acciones, el monitoreo de la cobertura informativa de los medios en los procesos electorales.

Con base en el trabajo de miles de voluntarios, incorporados en los diversos programas que el organismo desarrolla, y con el financiamiento de organizaciones internacionales, TRANSPARENCIA ha realizado el monitoreo de medios de la cobertura informativa de los procesos electorales recientes. ${ }^{3}$

Por su parte, la Veeduría Ciudadana de la Comunicación Social, organismo de la sociedad civil en la que confluyen asociaciones civiles,

\footnotetext{
${ }^{2}$ Revista Peninsular, semanario de información y análisis político núm. 558, junio 30 de 2000.

${ }^{3}$ Datos de la página Web de la asociación: http: / / www.transparencia.org.pe/
} 
académicas y eclesiásticas (Asociación de Comunicadores Sociales Calandria; Asociación Peruana de Consumidores y Usuarios, ASPEC; Asociación Peruana de Facultades de Comunicación Social, APFACOM; Comisión Episcopal de Comunicación Social, canamcos; Defensoría del Pueblo; Foro Educativo; Instituto de Defensa Legal, IDL; Asociación Civil Transparencia) destaca como su principal interés el establecimiento de "puentes cualitativamente mejores entre audiencias y medios", que repercutan en "la formación de mejores ciudadanas y ciudadanos", contempla entre sus actividades la realización de observación de la oferta de los medios y de su comportamiento ante diversas coyunturas, entre las cuales destacan los procesos electorales. ${ }^{4}$

Durante las elecciones presidenciales de 2000, ambos organismos desarrollaron una intensa actividad de observación electoral, entre las que se encuentra la realización de un extenso y documentado monitoreo de las coberturas informativas de las campañas presidenciales que los diversos medios realizaron, en un contexto de efervescencia política provocado por la postulación de Fujimori a un tercer mandato, cuyo gobierno evidenciaba un importante desgaste, así como el sometimiento de los medios de comunicación de masas, a quienes su régimen autoritario y corrupto había impuesto una política de silenciamiento ante toda manifestación crítica de la sociedad civil (Carrillo s/f).

En Colombia, en las elecciones presidenciales de 1998, conforme a lo establecido en el artículo 27 de la ley 130 de 1994, que obliga a "los concesionarios de los noticieros y programas de opinión en la televisión a garantizar el pluralismo, el equilibrio informativo y la imparcialidad durante la campaña electoral', la Comisión Nacional de Televisión realizó un seguimiento a los noticieros televisivos con el objeto de "supervigilar el cumplimiento de esta norma" (Jaramillo y Franco-Cuervo 1999). Por su parte, la Veeduría Ciudadana surgida de la Comisión Ciudadana de Seguimiento al Juicio del Presidente Samper, "contrató un estudio de imparcialidad de 20 medios de comunicación, el cual cubrió el periodo comprendido entre el 20 de abril y el 21 de junio de 1998".

En Chile, las primeras experiencias de monitoreo de medios datan del año 2001. Con el financiamiento de la Fundación Konrad Adenauer, la Universidad del Pacífico y Ministerio Secretaría General de Gobierno, la asociación PARTICIPA, "Corporación privada con fines públicos, pluralista y no partidaria que propicia la creación de una ciudadanía

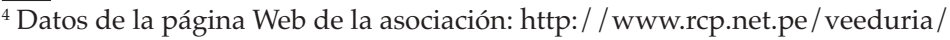
index.html
} 
informada, organizada, responsable y participativa", con el propósito de "promover un voto informado y de fortalecer el rol social de los medios de comunicación en el marco de las Elecciones Parlamentarias del 2001", realizó un monitoreo a 12 medios de comunicación en los que analizaron la cobertura informativa otorgada a candidatos, partidos y coaliciones políticas.

Más recientemente, con la asesoría de PARTICIPA de Chile y el financiamiento de la Konrad Adenauer, dos nuevos países del cono sur incorporaron, como parte de la observación ciudadana de los procesos electorales, el monitoreo de medios de comunicación masiva. En Paraguay, el Instituto de Derecho y Economía Ambiental de Paraguay (IDEA), con el objetivo de "contribuir a que la ciudadanía cuente con información veraz, equitativa y pertinente respecto de los candidatos y de las ofertas políticas de la campaña electoral", está realizando en el marco de las elecciones generales del 27 de abril de 2003, un monitoreo de medios para detectar las características de la cobertura informativa que le otorgan a las diferentes fuerzas políticas. De esta manera, IDEA busca contribuir "a decisiones de voto informadas y a promover el control ciudadano respecto al tratamiento que reciben los candidatos/as en periodo de campañas políticas en los medios de comunicación". ${ }^{5}$ Por su parte, en Argentina Poder Ciudadano, fundación apartidaria, constituida a finales de 1989 por iniciativa de un grupo de personas preocupadas por la defensa de los derechos cívicos de su país, realiza en el marco de su campaña "Iluminemos el cuarto oscuro" un monitoreo de medios de comunicación sobre la cobertura informativa de las campañas políticas, en el proceso electoral de 2003, cuyo objetivo central es el de "generar información rigurosa y oportuna que permita mejorar la calidad del debate público durante el periodo electoral, a fin de hacerlo más inclusivo, y promover que la ciudadanía acceda a información periodística lo más plural posible sobre la oferta electoral, a través de los medios de comunicación". ${ }^{6}$

Una iniciativa novedosa en el campo del monitoreo de medios, particularmente por el uso de los recursos del ciberespacio, es el programa de e-Voluntariado para Monitoreo de Medios impulsado por Reforma Política para la República (PRP), "grupo apartidario de ciudadanos" entre cuyos propósitos destacan el de "generar [una] opinión pública informada que logre impulsar la reforma política necesaria para salir de la

\footnotetext{
${ }^{5}$ Datos de la página Web de la asociación: http:/ www.idea.org.py/

${ }^{6}$ Datos de la página Web de la asociación: www.poderciudadano.org.ar/
} 
situación actual e ingresar en un círculo virtuoso en la vida de nuestras instituciones". El objetivo del programa es captar "artículos de opinión y noticias relevantes, orientados a elevar el debate y formar opinión pública acerca de una reforma política de fondo para la Argentina", actividad desarrollada por voluntarios cuyo compromiso consiste en "destinar tiempo personal a la búsqueda de noticias relevantes en los medios de difusión masiva y otras fuentes, e ingresarlas en el sistema de monitoreo mediante una interfase simple especialmente diseñada a tal fin".?

\section{EL IMPACTO DE LA OBSERVACIÓN CIUDADANA EN LA COBERTURA INFORMATIVA DE LOS MEDIOS EN LOS PROCESOS ELECTORALES}

No obstante su evidente contribución al desarrollo de elecciones democráticas, caracterizadas por un acceso a la información de mayor calidad para los ciudadanos y la promoción de condiciones de competencia electoral más equitativas, la práctica del monitoreo de medios de comunicación ha sido objeto de críticas y cuestionamientos.

Básicamente, se cuestiona el que su realización ponga en riesgo la libertad de expresión, en especial la referida a la libertad de prensa. A manera de ilustración, valgan las palabras del subdirector general de un diario capitalino:

[...] es injusto calificar el balance de las coberturas durante las campañas. Los pesos relativos de los partidos no son una constante, y menos en la etapa inmediatamente preelectoral. Una normatividad estricta para la medición apuntaría a la esclerotización de la percepción social de los partidos y pondría trabas, demostradamente innecesarias, a la libertad de expresión.

Junto con esto, está el asunto de la medición cualitativa de la información, que es algo notoriamente más difícil... ¿Quién garantiza la objetividad del "garante" (del fiscal)? ¿Quién dice si un encuadre es favorable, desfavorable o neutro? ¿Es permisible que, en aras de la paridad, haya intervenciones ajenas a los medios para guiar su línea editorial? ¿Hasta dónde se podrían tensar las garantías del artículo 6 de la Constitución? (Báez 1999).

La objeción se centra no tanto en la realización de los monitoreos, sino en el hecho de que se lleven a cabo durante el proceso electoral. Y

${ }^{7}$ Datos de la página Web de la asociación: www.reforma-politica.com.ar/ 
por ende, que sus resultados se difundan en el entorno de la contienda. Se trata evidentemente de una reacción defensiva frente al ejercicio de una acción de supervisión y vigilancia ciudadana al desempeño de las labores periodísticas. Más allá de la defensa a ultranza de la libertad de prensa, la declaración revela el temor a la sanción moral por parte de los usuarios de los medios.

Otro de los cuestionamientos a que se enfrenta la práctica de los monitoreos, tiene que ver con el alcance de su actividad, específicamente su impacto en el comportamiento informativo de los medios masivos. Es decir, si el monitoreo queda limitado a la ilustración de las condiciones mediáticas en que se desarrollan las campañas políticas, o si mediante su ejercicio de vigilancia logra incidir en la definición de las políticas editoriales de los medios y, por ende, en su comportamiento informativo.

Un análisis diacrónico de los monitoreos realizados, ofrece un testimonio elocuente del impacto de los monitoreos sobre los medios de comunicación. En el caso de México, tomando en cuenta exclusivamente factores cuantitativos, las diferencias observadas en el comportamiento de los telenoticieros en el lapso transcurrido entre los procesos electorales de 1988 y 2000, es por demás elocuente. En efecto, de una cobertura en extremo desigual que exhibieron los telenoticieros en 1988, cuando otorgaron al candidato del Partido Revolucionario Institucional (PRI) el 91.7\% del espacio total que dedicaron a las campañas presidenciales, en contraste con el $3.4 \%$ y $1.8 \%$ otorgado a los candidatos del Partido Acción Nacional (PAN) y del Frente Democrático Nacional (FDN) respectivamente, ${ }^{8}$ pasaron a una drástica modificación en sus patrones de cobertura en las elecciones de 1994, al disminuir a una tercera parte el tiempo concedido al candidato oficial e incrementar sustancialmente, aunque aún con rasgos de inequidad, la cobertura informativa de los principales contendientes políticos. Y aunque en el año 2000 el grueso de los telenoticieros aumentaron el tiempo de cobertura del candidato del PRI -que a la postre resultó perdedor- hasta $39.9 \%$, el candidato de la Alianza por el Cambio obtuvo $25.9 \%$ y el de Alianza por México ${ }^{9} 19.9 \%{ }^{10}$ (véase Tabla 1 ).

${ }^{8}$ Carlos Salinas de Gortari era el candidato del PRI, Manuel Clouthier del PAN y Cuauhtémoc Cárdenas del FDN.

${ }^{9}$ La Alianza por el Cambio se formó de una coalición entre el PAN y el Partido Verde Ecologista de México (PVEM); por su parte, la Alianza por México se integró de una coalición de partidos en torno al Partido de la Revolución Democrática.

${ }^{10}$ Los candidatos presidenciales en las elecciones de 2000 eran Francisco Labastida Ochoa (PRI), Vicente Fox (Alianza por el Cambio) y Cuauhtémoc Cárdenas (Alianza por México). 
TABLA 1

Cobertura INFORMativa DE LAS ELECCIONES PRESIDENCIALES (\%)

TELENOTICIEROS

\section{Difusión nacional}

\begin{tabular}{lllccr} 
& PAN/AC & PRI & FDN/PRD/AM & PVEM & Otros \\
$1988^{*}$ & 3.4 & 91.7 & 1.8 & - & 3.1 \\
$1994 * *$ & 15.3 & 32.0 & 18.3 & 8.9 & 25.5 \\
$2000 * * *$ & 25.9 & 39.9 & 19.9 & - & 14.3 \\
\hline
\end{tabular}

Fuente: 1988: Así se calló el sistema. Comunicación y elecciones en 1988, Universidad de Guadalajara, México, 1991 (*24 Horas y Día a Día).

1994 y 2000: Instituto Federal Electoral (**8 telenoticieros y ${ }^{* * *} 8$ telenoticieros).

Esta tendencia, que se observa a nivel nacional, se refleja también en el análisis de los monitoreos realizados por la Universidad de Guadalajara para el Consejo Electoral del Estado de Jalisco en los procesos electorales de 1995 y 2000. En este análisis es posible advertir una modificación drástica en los patrones de comportamiento de cobertura informativa de las campañas políticas otorgadas a los diversos candidatos. En todos los casos (prensa escrita, radionoticieros y telenoticieros) se aprecia una notable disminución del espacio otorgado al candidato del PRI, al mismo tiempo que un incremento del tiempo concedido a los candidatos de los otros partidos.

Esta modificación es particularmente notable en el caso de la prensa escrita (Tabla 2) en donde se aprecia que, de un $85.5 \%$ del tiempo dedicado a las campañas que se otorgó en 1989 al candidato del PRI, descendió a $66.5 \%$ en las elecciones de 1995 y a $34.7 \%$ en las del año 2000. Es necesario apuntar que en las elecciones de 1995, después de 65 años de gobernar el estado, a pesar de contar con una cobertura informativa claramente favorable, el candidato del PRI fue derrotado por un casi desconocido candidato del PAN. Y resulta notable el hecho de que en las elecciones de 2000, los candidatos de los dos partidos (PAN y PRI) que se dividen la mayoría del voto ciudadano, recibieron una cobertura que, en términos cuantitativos, podría calificarse de estricta equidad, al obtener 33.1 y $34.7 \%$ del espacio, respectivamente.

En el caso de los noticieros radiofónicos, aunque se observa en las modificaciones una tendencia a favorecer en ambos procesos al candidato del partido en el poder, la diferencia entre los candidatos de los partidos mayoritarios se reduce en las elecciones de 2000 respecto a las de 1995. 


\section{TABLA 2}

COBERTURA INFORMATIVA EN LAS ELECCIONES ESTATALES EN JALiSCO (\%) PRENSA ESCRITA

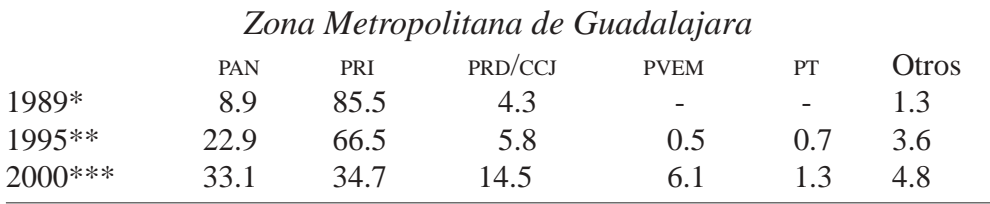

Fuente: 1989: Fregoso Peralta 1991. ( ${ }^{*}$ El Informador, El Occidental y Ocho Columnas). 1995: Seguimiento de la cobertura del proceso electoral 1995, Departamento de Estudios de

la Comunicación Social (**El Informador, El Occidental, Ocho Columnas y Siglo 21). 2000: Monitoreo de medios de comunicación en las elecciones estatales de 2000. Informe final, DeCs/CEej. ( ${ }^{* * *}$ El Informador, El Occidental, Ocho Columnas, Público, Mural).

\section{TABLA 3}

CobertuRA INFORMATIVA EN LAS ELECCIONES ESTATALES EN JALisCo (\%) RADIONOTICIEROS

\begin{tabular}{lllllll}
\multicolumn{8}{c}{ Zona Metropolitana de Guadalajara } \\
& PAN & PRI & PRD & PVEM & PT & Otros \\
$1995^{*}$ & 23.1 & 49.1 & 16.4 & 0.6 & 3.6 & 7.3 \\
$2000 * *$ & 37.8 & 29.9 & 15.3 & 0.6 & 0.5 & 4.6
\end{tabular}

Fuente: 1995: Seguimiento de la cobertura del proceso electoral 1995, Departamento de Estudios de la Comunicación Social (*Notisistema, XEDK, Radio Centro, Triple AAA y Radio Universidad).

2000: Monitoreo de medios de comunicación en las elecciones estatales de 2000. Informe final, DECS/CEEJ (** Resumen Informativo AAA, En Directo, Hoy por Hoy, Frecuencia Punto 3, Reporte en Vivo, En sintesis y Notisistema).

Finalmente, respecto a la cobertura realizada por lo noticieros televisivos, es posible advertir que las modificaciones en el patrón de cobertura entre ambos procesos ha sido drástica. Al igual que la prensa, en términos cuantitativos el comportamiento informativo de las empresas televisoras sufre una transformación radical, ya que no sólo distribuye un porcentaje similar de su espacio otorgado a la cobertura de las campañas de los candidatos de los partidos mayoritarios: $17.7 \%$ PAN y $16.8 \%$ PRI, sino que de todos los medios informativos, es el único que incluyó en su cobertura de campañas al grueso de los partidos políticos.

Pero tal vez el ejemplo más notable de la potencialidad del monitoreo como instrumento de vigilancia ciudadana y como herramienta para la promoción de procesos electorales democráticos, lo ilustra el caso de las 


\section{TABLA 4}

Cobertura INFORMATIVA EN LAS ELECCIONES ESTATALES EN JALISCO (\%) TELENOTICIEROS

\begin{tabular}{lllllll} 
& \multicolumn{6}{c}{ Zona Metropolitana de } \\
& PAN & PRI & PRD & PVEM & PT & Otros \\
$1995^{*}$ & 26.7 & 48.8 & 9.9 & 4.8 & - & 9.8 \\
$2000^{* *}$ & 17.7 & 16.8 & 10.8 & 6.1 & 6.5 & 36.6 \\
\hline
\end{tabular}

Fuente: 1995: Seguimiento de la cobertura del proceso electoral 1995, Departamento de Estudios de la Comunicación Social ( ${ }^{*}$ Canal 4 y Canal 6).

2000: Monitoreo de medios de comunicación en las elecciones estatales de 2000. Informe final, DECS/CEEJ.

(**Buenos Días Guadalajara, Esta Mañana Jalisco, Impulso Matutino, Hechos Jalisco, Hoy por Hoy, Al Tanto y Antena 5).

elecciones para la gubernatura en Tabasco en el año 2000, cuando la denuncia presentada por los grupos de observación electoral acerca de la inequidad en el tratamiento informativo que la televisora estatal (TVT) había realizado de las campañas políticas, al otorgar una cobertura descaradamente preferencial al candidato del Partido Revolucionario Institucional, fue considerada por el Tribunal Electoral del Poder Judicial de la Federación como uno de los principales argumentos en los que basó su decisión para determinar la anulación del proceso. ${ }^{11}$

\section{Conclusiones}

Los monitoreos de medios constituyen una herramienta eficaz de vigilancia y control social del comportamiento informativo de los medios de comunicación para garantizar una participación más y mejor informada de la ciudadanía en las decisiones electorales, así como el establecimiento de condiciones de equidad en el acceso a los medios de comunicación de los diversos actores políticos y, de esta manera, promover el desarrollo de procesos electorales democráticos.

Resulta entonces fundamental, para el avance democrático en América Latina, establecer la práctica de monitoreo de medios para la vigilancia de la cobertura informativa de los procesos electorales en todos y cada uno de los países. La participación de organismos surgidos de la sociedad civil es sumamente valiosa; sin embargo, habría que pugnar

\footnotetext{
$\overline{11}$ "Fue inequitativa: Lucio Lastra, 'La clave fue TVT'”, por José Antonio Ruiz
} Estrada, México Hoy, diciembre de 2000. 
para que en las legislaciones electorales de los respectivos países se estableciera, como obligación de las autoridades electorales, la realización de tales monitoreos y la difusión pública de sus resultados durante y a lo largo del proceso electoral. En forma paralela, pugnar por la promulgación de preceptos legales, con rango constitucional, que subordinen los intereses particulares de los concesionarios de los medios masivos de comunicación a las necesidades sociales de derecho a la información y al desarrollo democrático de nuestras sociedades.

\section{REFERENCIAS BIBLIOGRÁFICAS}

Aceves GonzÁlez, Francisco de Jesús (2001) Los medios de comunicación y el electorado tapatío en las elecciones presidenciales de 1994, tesis doctoral, Universidad de Guadalajara.

Acosta ValVerde, Miguel (1997) Los medios de comunicación y la educación ciudadana. México: AMDH.

, y Luz Paula Parra Rosales (1995) Los procesos electorales en los medios de comunicación. Guía para el análisis de contenido electoral en México. México: AMDH-Universidad Iberoamericana.

Álvarez Icaza, Emilio (s/f) "Medios y sociedad civil: una alianza para el control político", Razón y Palabra, núm 18. www.razonypalabra.org.mx/anteriores/n18/

BÁEZ, Francisco (1999) "Intervención de los medios en los procesos electorales", ponencia en el Seminario Internacional Partidos Políticos, Medios de Comunicación y el Proceso Democrático, IFE, 3 y 4 de febrero.

Carrillo, Sonia Luz (s/f) "Actuación política de los medios de comunicación peruanos. Elecciones generales del 2000 y 2001”, Razón y Palabra, núm. 26. www.cem.itesm.mx/dacs/publicaciones/logos/anteriores/n26/sluz.html

De Garay, Adrián, y Laura Moya López (1989) “Aproximaciones al estudio de la opinión pública y la prensa: las campañas electorales de 1988”, Sociológi$c a$, año 4, núm. 11, septiembre-diciembre. México: UAM-Azcapotzalco.

Fregoso Peralta, Gilberto (1990) "Las elecciones federales de 1988 en la prensa de Guadalajara", Comunicación y Sociedad, núm. 8, enero-abril. Guadalajara: CeIc-Universidad de Guadalajara. 
(1991) "Cinco meses después...", Así se calló el sistema. Guadalajara: Universidad de Guadalajara.

JarAmillo Pérez, Juan, y Beatriz Franco-Cuervo (1999) "Las elecciones presidenciales en Colombia 1998", en Frank Priess y Fernando Tuesta (eds.), Campañas electorales y medios de comunicación en América Latina, Tomo I. Argentina: Konrad Adenauer Stiftung/CIEDLA.

MAtus LóPEZ, Juliana (2001) "Monitoreo de prensa en el proceso estatal electoral de Chiapas 2000”, Revista Latina de Comunicación Social, año 4, núm. 39, marzo, La Laguna (Tenerife).

Norris, Robert, y Patrick Merloe (2002) Media Monitoring to Promote Democratic Elections. An NDI Handbook for Citizen Organizations. Washington DC: National Democratic Institute for International Affairs (NDI).

Secanella, Petra María (1983) El periodismo político en México. Barcelona: Ediciones Mitre.

Trejo Delarbre, Raúl (1990) "Campaña y elecciones en la prensa de la ciudad de México", Comunicación y Sociedad, núm. 8, enero-abril. Guadalajara: CEIC-Universidad de Guadalajara. También en Así se calló el sistema, Universidad de Guadalajara. 